\title{
Decorin Inhibits Glucose-induced Lens Epithelial Cell Apoptosis via Suppressing Rac1-p38 MAPK Signaling Pathway
}

\author{
Jingzhi Shao ${ }^{1,2}$, Shanshan Du ${ }^{1}$, Jingjing Wan ${ }^{1}$, Lirong Zhang ${ }^{2}$, Fengyan Zhang ${ }^{1,}$, \\ ${ }^{1}$ Department of Ophthalmology, the First Affiliated Hospital of Zhengzhou University, Henan Province Eye Hospital, Zhengzhou, China \\ ${ }^{2}$ Department of Pharmacology, School of Basic Medical Sciences, Zhengzhou University, Zhengzhou, China
}

Email address:

shaozi402@163.com (Jingzhi Shao), fcczhangfy@zzu.edu.cn (Fengyan Zhang)

${ }^{*}$ Corresponding author

\section{To cite this article:}

Jingzhi Shao, Shanshan Du, Jingjing Wan, Lirong Zhang, Fengyan Zhang. Decorin Inhibits Glucose-induced Lens Epithelial Cell Apoptosis via Suppressing Rac1-p38 MAPK Signaling Pathway. International Journal of Ophthalmology \& Visual Science. Vol. 6, No. 1, 2021, pp. 1-9. doi: $10.11648 / j . i j o v s .20210601 .11$

Received: January 13, 2021; Accepted: January 27, 2021; Published: January 30, 2021

\begin{abstract}
Aim This study was conducted to evaluate the influence of decorin on apoptosis and oxidative stress response upon exposure of human lens epithelial (HLE) cells to high levels of glucose. Methods The HLE cell line (HLEB3) was cultured under various conditions, including normal $(5.5 \mathrm{mM})$ or high glucose $(60 \mathrm{mM})$ medium. Next, $50 \mathrm{nM}$ Decorin was added two hours prior to the addition of high glucose medium. Apoptosis detection was carried out by Western blotting and flow cytometry. Oxidative stress levels were determined by quantifying reactive oxygen species (ROS), superoxide dismutase (SOD), and glutathione peroxidase (GSH) levels. P38 mitogen-activated protein kinase (MAPK) phosphorylation and rac1 in HLE cells and human lens anterior capsules were evaluated using Western blotting. Knockdown of rac1 and p38 MAPK in HLEB3 cells was achieved using transfection of small-interfering RNAs. Results HLE cells exposed to high levels of glucose underwent oxidative stress and apoptosis, leading to higher proportion of apoptotic cells, ROS generation and a reduction in the bcl/bax ratio, GSH/GSSG ratio, and SOD activity. Additionally, HLEB3 cells exposed to high levels of glucose were found to have increased rac1 and phospho-p38 MAPK. Next, we found that siRNA-mediated knockdown of rac1 or p38 led to a reduction in high-glucose-stimulated cellular apoptosis and oxidative stress. Furthermore, knockdown of rac1 led to a downregulation of p38 MAPK. Interestingly, addition of decorin to HLEB3 cells led to a decrease in apoptosis, oxidative stress, and high-glucose-stimulated induction of rac1 and phospho-p38. Finally, rac1 and p38 levels of capsules were detected to be significantly upregulated in patients with diabetes in comparison to age-matched patients with cataracts. Conclusion The findings indicate that the rac1-p38 pathway plays a role in causing high-glucose-induced injury to the lens epithelial cells. Additionally, these data indicate that decorin can suppress high glucose-stimulated cellular apoptosis and oxidative stress, in part through suppression of the rac1-p38 pathway.
\end{abstract}

Keywords: High Glucose, Decorin, Apoptosis, Oxidative Stress

\section{Introduction}

Diabetic cataract, which is frequently seen in clinic, is a significant diabetes-associated complication [1]. Highglucose-induced oxidative stress is vital to diabetic cataract development. High levels of glucose cause apoptosis, oxidative stress and aggregation of proteins. In turn, oxidation and aggregation of proteins lead to lens opacity [2]. Apoptosis and oxidative stress tend to occur when human lens epithelial (HLE) cells are incubated with high glucose [3].
The leucine-rich proteoglycan decorin is known to bind to the extracellular matrix (ECM) or cell surface receptors, and negative controls several cellular functions [4, 5]. Overexpressed decorin can inhibit tumor cell-regulated angiogenesis by suppressing endogenous vascular endothelial growth factors (VEGFs) production [6]. Administering decorin into the corneal stroma inhibits neovascularization of the cornea in an animal model of disease [7]. Additionally, studies reported that decorin is able to hinder oxidative stress-related injury and apoptosis in the cerebrum of a 
post-traumatic brain injuries (TBI) rat model [8, 9]. Our preliminary study showed that decorin inhibits retinal pigment epithelial (RPE) barrier disruption in the context of diabetes by hindering activation of $\mathrm{p} 38$ mitogen-activated protein kinase (MAPK) [10]. Nevertheless, how decorin influences the development of diabetic cataracts remains unclear.

In this study, we evaluated the efficacy and possible mechanism of decorin on cellular apoptosis and oxidative stress upon exposure to high levels of glucose and the therapeutic potential of decorin on HLE cells was evaluated. Apoptosis, reactive oxygen species (ROS), superoxide dismutase (SOD), and glutathione peroxidase (GSH) were examined. In addition, p38 MAPK phosphorylation and expression of rac1 in HLEB3 cells and human lens anterior capsules were examined. Additionally, we explored the function of rac1 and p38 MAPK in modulation of oxidative stress under high levels of glucose.

\section{Materials and Methods}

\subsection{Antibodies and Chemical Reagents}

Anti-bcl-2, anti-bax, anti-rac1, anti-p38 and anti-phospho-p38
(Thr180/Tyr182) were purchased from Abcam. Anti- $\beta$-actin, Anti-mouse and Anti-rabbit antibodies were bought through Proteintech. The recombinant human decorin was bought through R\&D System. The cell counting Kit 8 (CCK-8) and apoptosis detection kits were purchased from Beyotime Inst. Biotech.

\subsection{Human Lens Anterior Capsules}

Human lens anterior capsular tissues were acquired through the eye tissue bank located at the First Affiliated Hospital of Zhengzhou University. Protocols collecting the human lens anterior capsules were in accordance with guidelines for acquisition of human tissues. The protocols were granted approval by the Ethics Committee at the First Affiliated Hospital of Zhengzhou University. We acquired written informed consent from all participants. The participants did not receive any particular treatments. Overall, 27 patients with cataracts and type 2 diabetes, comprising of 30 eyes, were designated as the diabetic cataract group. On the other hand, 25 cataract patients that did not have systemic or ocular disease (control group), comprising of 30 eyes, were classified as the senile cataract group (Table 1).

Table 1. Cataract Patients Data.

\begin{tabular}{llll}
\hline & Diabetic Cataract Patients & Senile Cataract Patients \\
\cline { 2 - 4 } & Mean \pm SD & Range & Mean \pm SD \\
\hline Sample size (M:F) & $27(14: 13)$ & & $25(13: 12)$ \\
Eye number (R:L) & $30(16: 14)$ & $0.25-25$ & $30(13: 17)$ \\
Diabetes, yr & $10.67 \pm 4.97$ & $45-90$ & $64.64 \pm 11.08$ \\
Age, yr & $65.37 \pm 9.37$ & $1-4$ & $2.80 \pm 0.71$ \\
Cataract grade & $2.80 \pm 0.66$ & $7-10$ & $48-91$ \\
Hemoglobin A1c, \% & $8.64 \pm 0.96$ & $3.98-8.98$ & $1-4$ \\
Blood glucose & $7.32 \pm 1.26$ & & \\
Receive treatment & $100 \%$ & & \\
\hline
\end{tabular}

Standard deviation (SD); male (M); female (F); year (yr); Blood glucose, fasting blood glucose on the day of operation.

\subsection{Human Lens Epithelial Cell Cultures}

HLEB3 was maintained at $37^{\circ} \mathrm{C}$ in $5 \% \mathrm{CO}_{2}$ and cultured in complete media (DMEM with $5.5 \mathrm{mM}$ D-glucose (Sigma, USA), $10 \%$ FBS (Genial, USA) and 1\% antibiotics (Solarbio, China)). Cells were passaged when cell density approached $80 \%$.

HLEB3 cells were placed into six-well plates in complete media. When cell confluency reached about 50\%, 1\% FBS was added for a period of 12 hours. Next, cells were separated into four cohorts including the control (complete media), high glucose (60 mM D-glucose), decorin and mannitol group. The control group was cultured normally. The high glucose group was exposed to $60 \mathrm{mM}$ glucose for a period of 72 hours. The decorin group was treated using glucose 72 hours after being treated with $50 \mathrm{nM}$ recombinant human decorin for two hours [11]. The mannitol group was treated with $54.5 \mathrm{mM}$ mannitol in order to eliminate variation by osmosis.

\subsection{Cell Viability Assay}

CCK-8 assay was utilized to evaluate cell viability. Cells were cultured overnight in 96 -well plates at $1 \times 10^{4}$ cells/well density. Next, new media with $0,50,100$, and $200 \mathrm{nM}$ of decorin was added, respectively. After a 24 hours incubation, $10 \mu \mathrm{l}$ of WST-8 [2-(2-methnoxy-4-nitrophenyl)-3-(4-nitrophenyl)-5-(2,4-disulfophenyl)-2H-tetra-zolium, sodium salt] (Beyotime, China) was placed in every well. Then, the plates were kept in a $37^{\circ} \mathrm{C}$ incubator for another four hours. After that, plates were read at $450 \mathrm{~nm}$ through the use of iMark $^{\mathrm{TM}}$ microplate absorbance reader (Bio-Rad, Hercules, USA). The amount of live cells within every well is represented in relation to controls.

\subsection{Small Interfering RNA (siRNA) Transfection}

HLEB3 cells were transfected with a mixture of $100 \mathrm{nM}$ siRNAs targeting rac1 or p38 MAPK (San Cruz Biotechnology, USA) or scramble control in accordance with the kit's instructions. Six hours post incubation with siRNAs, HLEB3 cells were cultured using high glucose, as previously described.

\subsection{Flow Cytometry}

Flow cytometry was utilized to measure cell apoptosis in 
accordance with Annexin-V-FITC apoptosis detection kit (BD Bioscience, USA) protocol. First, HLEB3 cells were gathered. Second, the cells were labelled with Annexin-V-FITC at room temperature for $10 \mathrm{~min}$. Third, stained cells were washed and centrifuged three times. Fourth, cells were resuspended, labelled with propidium iodide (PI) and underwent flow cytometry (BD ${ }^{\mathrm{TM}}$ LSR II ) (BD Bioscience, USA). Win MDI 2.9 software were used for analyzing data. Viable cells are FITC-Annexin V and PI double negative, while cells that exhibit the FITC-Annexin-V positive and PI negative are defined to be in early apoptosis. Cells that were stained double positive by FITC-Annexin-V and PI are apoptotic.

\subsection{Intracellular ROS Quantification}

Intracellular ROS, which was labelled using 2', 7'-dichlorofluorescein diacetate (DCFH-DA), was tested by the densitometry measuring of the DCFH-DA detection kit (Beyotime, China). DCFH-DA could be oxidized and transformed into fluorescent dichlorofluorescein (DCF) under oxidative stress condition. This can be measured with a BD Accuri C6 Flow Cytometer (Bioscience, USA). Briefly, after being collected, diluted DCFH-DA was added to HLEB3 cells for $20 \mathrm{~min}$, followed by washing with serum-free DMEM. After discarding the liquid supernatant resulting from centrifugation, cells were resuspended in PBS. Then, cellular fluorescence was quantified using $488 \mathrm{~nm}$ excitation and 525 $\mathrm{nm}$ emission wavelengths of the BD Accuri C6 Flow Cytometer.

\subsection{SOD and GSH Detection}

Activity of intracellular SOD, the GSH and GSSG were detected as per instruction manual provided by the total SOD assay kit and GSH/GSSG assay kit (Beyotime Institute, China), respectively. The plates treated by the kits were quantified through the use of iMark $^{\mathrm{TM}}$ microplate absorbance reader at $450 \mathrm{~nm}$ and $412 \mathrm{~nm}$, respectively.

\subsection{Western Blotting}

Western blotting was carried out according to previous studies [12]. Protein levels were detected through an enhanced chemiluminescence detection system (ProteinSimple, San Jose, California, USA). Band intensity was analyzed with Alpha View SA version 3.4.0 software (ProteinSimple).

In order to examine protein expression of human lens anterior capsules, we obtained the anterior capsules from patients that were subjected to cataract surgery. Overall, 10 anterior capsules had to be acquired from within every groups and then combined together to obtain adequate protein for immunoblotting analysis.

\subsection{Statistical Analysis}

Results from a minimum of three experiments were expressed as mean \pm standard deviation (SD). We utilized SPSS 18.0 software to perform all statistical analyses. A one-way analysis of variance (ANOVA) was utilized to compare data from more than two groups. Comparison within two groups were investigated through student-Newman-Keuls test. $P<0.05$ represents statistical significance.

\section{Results}

\subsection{Cell Viability Assay}

HLEB3 cell viability was examined with a CCK-8 assay with different concentrations of decorin $(0,50,100$ and 200 $\mathrm{nM})$. Results showed no significant differences within groups (Figure 1E). Moreover, obvious morphological changes were not observed at different concentrations of decorin with phase contrast microscopy (Figure 1A-D).
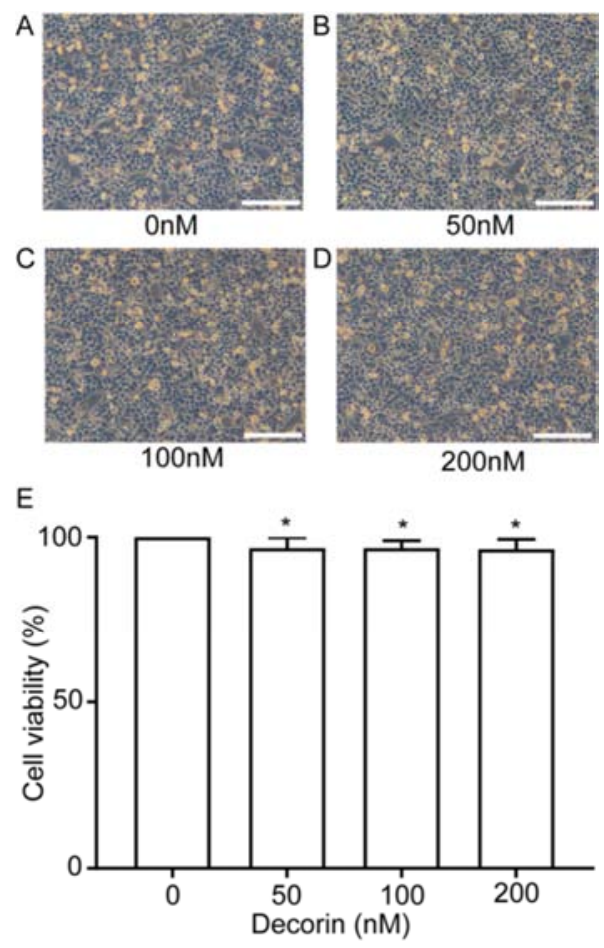

Figure 1. HLEB3 cell viability with different concentrations of decorin.

(A-D) Change in cell morphological appearance at various doses of decorin. No overt morphological changes were found between groups. (E) No significant cell viability differences were detected among groups when tested with a CCK- 8 assay ( ${ }^{*} P>0.05$ vs. $0 \mathrm{nM}$ group). Scale bars $=20 \mu \mathrm{m}$, data is represented as bars, which indicate mean $\pm \mathrm{SD}$.

\subsection{Decorin Suppresses HLEB3 Cell Apoptosis Stimulated Through High Glucose}

The effect of decorin on HLEB3 cellular apoptosis was depicted (Figure 2). First, cells were divided to four groups, including viable cells (unlabeled), early apoptotic cells (labelled using annexin V-FITC), necrotic cells (labelled using $\mathrm{PI}$ ), and late apoptotic cells (labelled using annexin V and PI). Apoptotic cells were defined as the sum of late and early apoptotic cells. The proportion of apoptotic cells in the control group was $5.1 \% \pm 0.50 \%$. Treatment with high levels of glucose heightened the proportion of apoptotic cells to $14.2 \%$ $\pm 0.46 \%(P<0.001)$. Cells that were administered $50 \mathrm{nM}$ decorin prior to high glucose treatment experienced a 
reduction in apoptosis to near normal levels (Figure 2A and B). Moreover, data from Western blot indicated that treatment with high glucose led to high bax expression and low bcl-2. Furthermore, according to densitometry analysis, the bcl-2 to
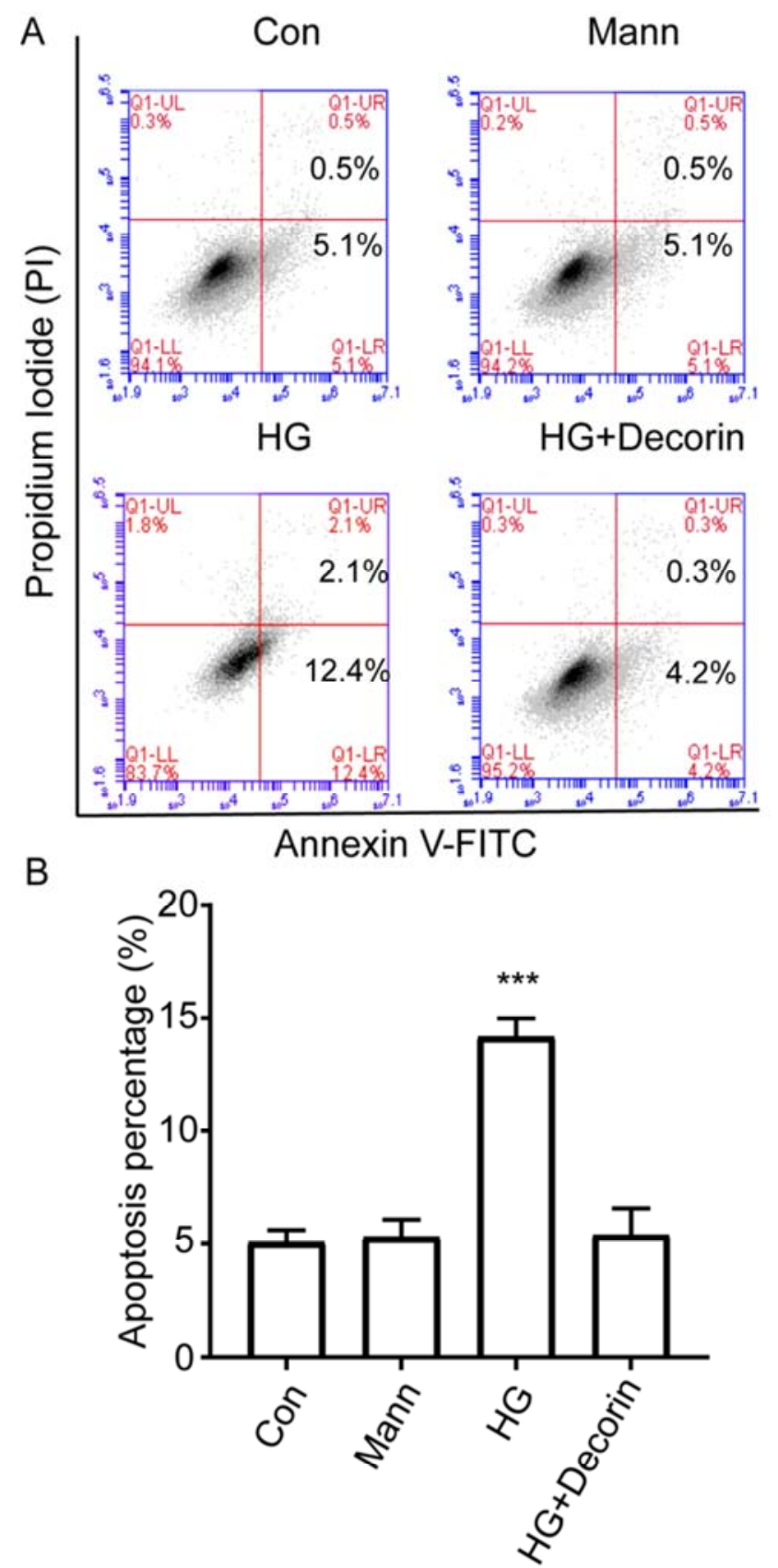

bax ratio was significantly lower within the high glucose group in comparison to controls. Under high-glucose conditions, decorin was able to reverse the bcl-2 to bax ratio (Figure 2C and D).

Figure 2. Effect of decorin on apoptosis of HLEB3.

(A) Quantitative cellular apoptosis analysis examined through flow cytometry using Annexin-V-FITC apoptosis staining. The upper-right quadrant represents late apoptotic cells, while the lower-right quadrant represents early apoptotic cells. (B) Total apoptotic cells were determined by addition of late and early apoptotic cells, and then statistically analyzed. (C) Bcl-2 and bax western blot in HLEB3 cells. (D) Bcl-2 to bax ratio, determined by optical density. Treatment with decorin decreased high glucose-stimulated apoptosis. Control (Con); Mannitol (Mann); high glucose (HG). Data represents mean $\pm \mathrm{SD}$. $* P<0.05$ represents significance in comparison to the control group.

\subsection{Decorin Suppresses High-glucose Stimulated Oxidative Stress of HLEB3 Cells}

Compared to controls, ROS production after administration of high-glucose was significantly higher. However, the proportion of GSH/GSSG and SOD activity were statistically reduced in HLEB3 cells. Decorin can suppress oxidative stress in HLEB3 cells exposed to high levels of glucose (Figure 3). 


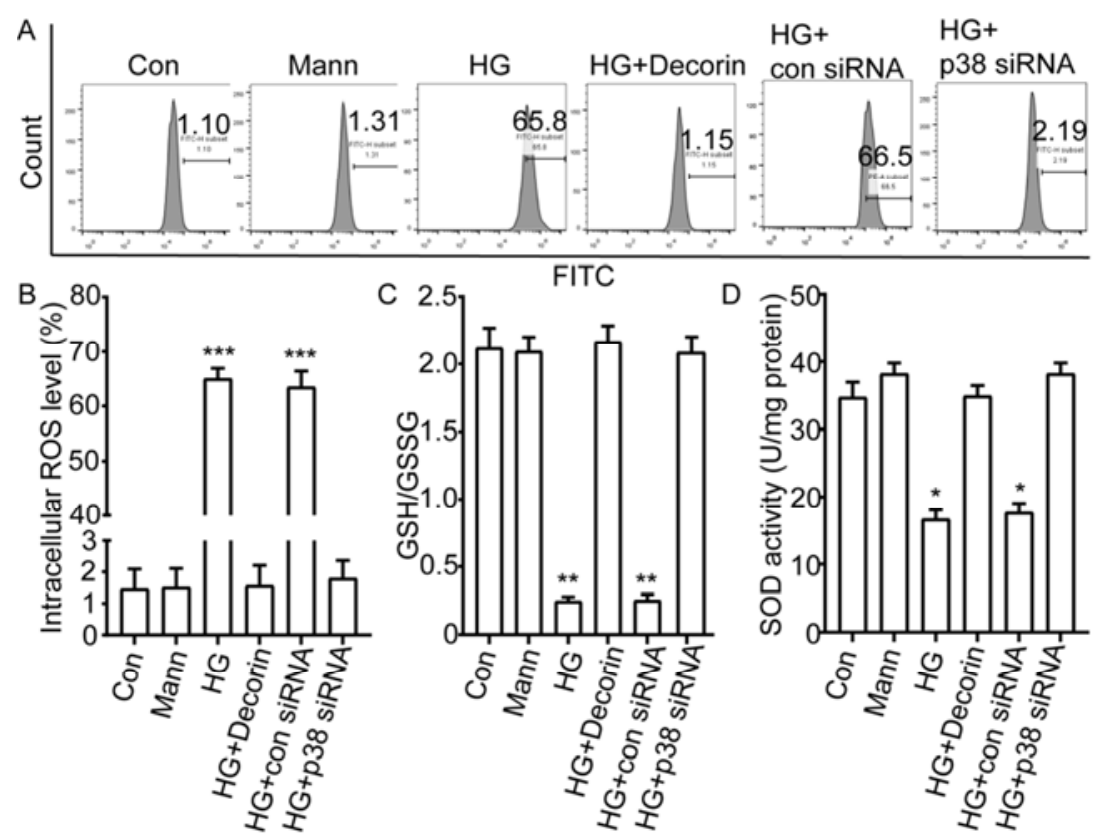

Figure 3. Intracellular oxidative stress alteration.

(A-B) Intracellular ROS levels were measured with a BD Accuri C6 Flow Cytometer. (C) The GSH/GSSG ratio is indicated for each group. (D) SOD activity in indicated groups. Decorin decreased the oxidative stress response stimulated by high levels of glucose. Control (Con); mannitol (Mann); high glucose (HG). Data are mean \pm SD. $* P<0.05, * * P<0.01, * * * P<0.001$, versus controls, respectively.

\subsection{Decorin Suppresses $p 38$ and Rac1 Activation in HLEB3 Cells Stimulated by High Levels of Glucose}

The activation of p38 and rac1 was detected with western blot. Under high-glucose conditions, densitometry analysis of p38 MAPK and rac1 showed significant increase in

A

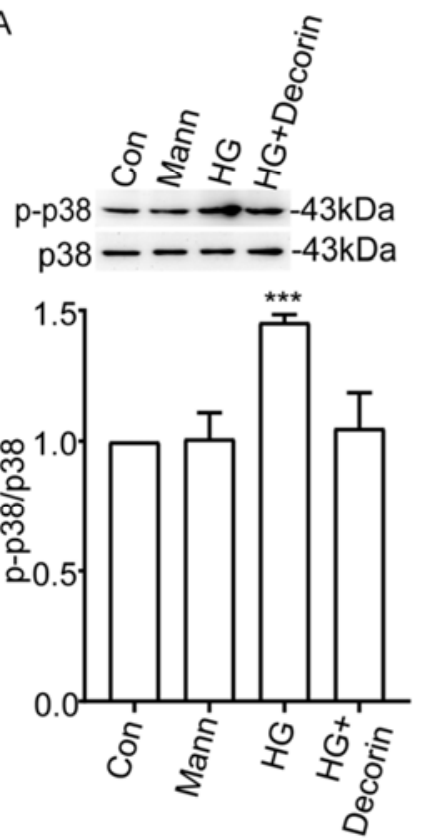

comparison to the control group. However, after being treated with $50 \mathrm{nM}$ decorin, p38 MAPK phosphorylation and rac1 protein was decreased significantly in comparison to the high-glucose group (Figure 4).

B

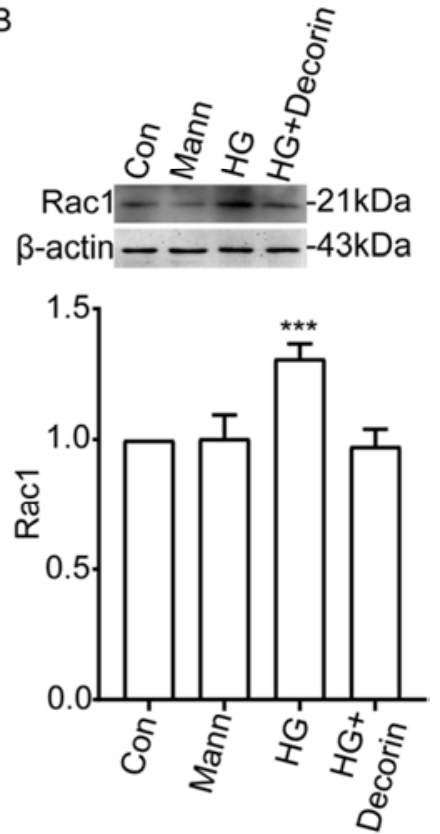

Figure 4. Influence of decorin on p38 phosphorylation and racl by high glucose in HLEB3 cells.

(A) Western blot of p38 MAPK phosphorylation. (B) Western blot of rac1. $\beta$-actin serves as internal control. Decorin decreased p38 MAPK activation and expression of rac1 in cells exposed to high glucose. Control (Con); mannitol (Mann); high glucose (HG). Data is represented as mean $\pm \operatorname{SD}$. $* * * P<0.001$ in comparison to the controls. 


\subsection{P38 MAPK Regulates Oxidative Stress and Apoptosis of HLEB3 Cells Stimulated by High Levels of Glucose}

To determine if p38 MAPK was associated with high-glucose-stimulated cellular apoptosis and oxidative stress, p38 MAPK siRNAs were transfected into HLEB3 cells. Next, p38 MAPK expression was examined using western blotting. As expected, p38 siRNAs significantly reduced p38 MAPK expression (Figure 5A). After siRNA transfection,

A
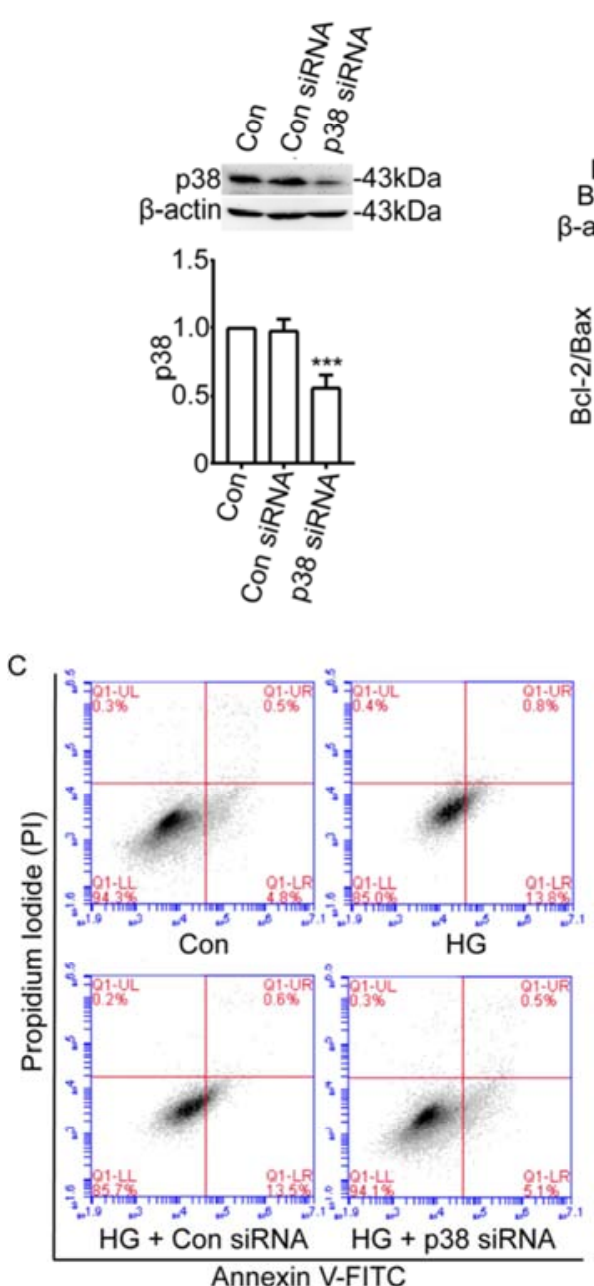

D

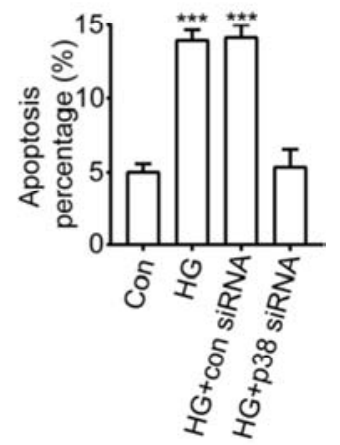

Figure 5. 38 MAPK siRNA inhibits HLEB3 cellular apoptosis stimulated by high glucose.

(A) p38 MAPK siRNA transfection led to a substantial decrease in p38 protein expression, while control siRNA did not have an effect. (B) Bcl-2 and bax western blot, and analysis of bcl-2 to bax optical density ratio. (C) Quantitative analysis of cellular apoptosis through flow cytometry. The upper-right quadrant exhibits cells undergoing late apoptosis, while the lower-right quadrant exhibits those under early apoptosis. (D) Apoptotic cells were defined as addition of late and early apoptotic cells. Proportion of apoptotic cells was statistically analyzed. Control (Con); high glucose (HG). Data is quantified as mean $\pm \mathrm{SD}$. ${ }^{*} P<0.05, * * * P<$ 0.001 vs. controls.

\subsection{High Glucose-Stimulated Cellular Apoptosis and Oxidative Stress May Be Mediated by the Rac1-p38 MAPK Pathway in HLEB3 Cells}

In order to evaluate the presence of a relationship among p38 MAPK and rac1 in regulating high glucose-stimulated oxidative stress, siRNAs targeting rac1 were transfected into HELB3 cells. Western blotting helped examine protein expression. As expected, rac1 expression was substantially decreased using rac1 siRNA (Figure 6A). Interestingly, rac1 siRNA transfection decreased p38 phosphorylation in high glucose-treated cells (Figure 6B). This suggests that rac1 regulates p38 MAPK activation and can help regulate high glucose-stimulated apoptosis and oxidative stress. 
A

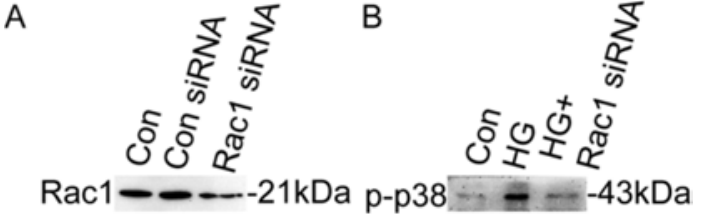

$\beta$-actin $=-43 \mathrm{kDa} \quad \mathrm{p} 38=--43 \mathrm{kDa}$

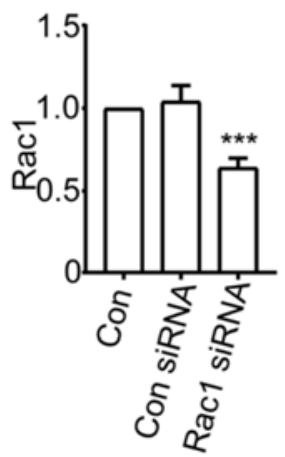

Figure 6. Possible relationship among $p 38 M A P K$ and racl in regulating high-glucose induced oxidative stress.

(A) Rac1 siRNA transfection led to a significant decrease in rac1 expression, while control siRNAs had no effect. (B) Rac1 siRNA transfection led to an obvious decrease in expression of phospho-p38 MAPK. Rac1 mediates p38 MAPK activation. Control (Con); high glucose (HG). Bars are indicative of mean $\pm \mathrm{SD}$, with $\beta$-actin as internal control. $* * * P<0.001$ vs. controls.

\subsection{Rac1 and Phospho-p38 Are Highly Expressed in Diabetic Lens Anterior Capsules}

We next evaluated rac1 and phospho-p38 MAPK expression in lens anterior capsules. Interestingly, we found that rac1 levels were substantially higher in the anterior capsules obtained through patients with diabetic cataracts compared to patients with simple senile cataract (Figure 7A). Similar results were obtained with regards to phospho-p38 expression (Figure 7B). These findings were consistent with those of the in vitro assay, and they further suggest that the rac1-p38 pathway likely participates in modulation of oxidative stress in the diabetic lens.

A
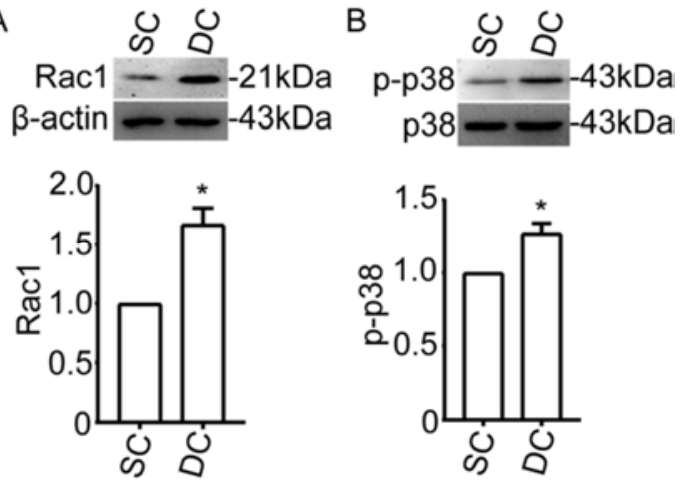

Figure 7. Racl and phospho-p38 expression in human lens anterior capsules.

(A) Rac1 immunoblotting analysis. (B) p38 MAPK immunoblotting analysis. Rac1 and phospho-p38 in the anterior capsules of patients with diabetic cataracts are significantly increased versus patients with simple senile cataracts. Senile cataract (SC); diabetic cataract (DC). $\beta$-Actin served as internal control. Data is exhibited as mean $\pm \mathrm{SD}$. ${ }^{*} P<0.05$ vs. SC group.

\section{Discussion}

Oxidative stress under high-glucose conditions is one of the main causes of diabetic cataracts [2]. The HLEB3 cell line has been widely used in research studies [13-15]. Data from our analysis indicates that high-glucose levels stimulate direct oxidative stress and cellular apoptosis of HLEB3 cells. P38 and rac1, which are vital signaling molecules in many pathways, participate in these processes. Rac1 works as an upstream modulator of p38 MAPK. Oxidative stress and cellular apoptosis under conditions of high glucose can be inhibited by decorin. Besides, high glucose-induced rac1 levels and p38 MAPK activation can be inhibited by decorin. These findings suggest that decorin can play a possible therapeutic function on the development of diabetic cataracts.

Rac1, a part of the superoxide-generating nicotinamide adenine dinucleotide phosphate (NAPDH) oxidase (NOX) complex, generates ROS within the mitochondria under conditions of oxidative stress [16]. Additionally, rac1, a small GTPase of the Rho family, has a key function in regulating several different cellular processes such as proliferation, apoptosis, migration, and invasion [17]. Furthermore, rac1 has an influence on diverse signaling pathways associated with $\mathrm{NF}-\kappa \mathrm{B}$, and JNK, and ROS production [18]. High expression of rac1B leads to ROS production [19]. Rac1 is upregulated in ARPE-19 cells under hypoxia [11]. David et al. indicated that impeding rac1 led to abolishment of phosphorylation of $\mathrm{p} 38$ MAPK signaling [20]. Rajakrishnan et al. showed that activation of $\mathrm{p} 38$ MAPK was downstream of rac 1 activation in diabetes-induced metabolic stress, and leads to capillary cell apoptosis [21]. Our data indicates that this signaling pathway is associated with injury to the diabetic lens epithelial cells. There are several lines of evidence to support this. First, high glucose induces ROS generation, downregulation of the GSH/GSSG ratio and inhibition of SOD activity, which causes increased apoptosis of HLEB3 cells. Second, high glucose induces rac1 and p38 MAPK activation. Silenced rac1 downregulates p38 MAPK activation stimulated by high glucose levels, and silenced p38 MAPK inhibits high glucose-stimulated ROS generation and cellular apoptosis. The idea of ROS as a vital stimulator or cellular apoptosis or necrosis in several types of tissues is widely accepted [22]. Third, phospho-p38 and rac1 were found to be highly expressed in the human lens anterior capsules of patients with diabetic cataracts in comparison to patients with senile cataracts. These findings indicate that rac1 regulates ROS generation through its downstream regulator, p38 MAPK, in lens epithelial cells under diabetic stress conditions.

The small leucine-rich proteoglycan, decorin, is a part of the extracellular matrix $[4,5]$. Additionally, decorin helps regulate angiogenesis, tumor proliferation, apoptosis and inflammatory processes [6-9]. Here, our results gave us some inspirations. First, in vitro treatment, decorin inhibits high-glucose-stimulated oxidative stress in lens epithelial cells. Second, administering decorin led to a decrease in ROS production, increased GSH/GSSG ratio, and increased the ratio of bcl-2 and bax in cells exposed to high levels of glucose, 
indicating that decorin can be used as a medical treatment to prevent the occurrence of cataract in diabetic patients. Third, in HLEB3 cells, decorin inhibits rac1 expression and p38 MAPK activation under conditions of high-glucose. In a prior study conducted by our lab, we explored the effect of decorin inhibition on the $\mathrm{p} 22^{\text {phox }}-\mathrm{p} 38$ MAPK pathway [23]. Results from that study showed that decorin protect HLEB cells by suppressing the p22phox-p38 MAPK pathway. The stimulation of p38 MAPK was downstream of rac1 and $\mathrm{p} 22^{\text {phox }}$ activation in diabetes-stimulated oxidative stress, leading to HLEB3 cellular apoptosis. From these studies, we can conclude that decorin has an effect on hindering diabetic cataract development by suppressing rac1-p38 MAPK pathway and p22 $2^{\text {phox }}$-p38 MAPK pathway.

In summary, our study demonstrated that high glucose induced oxidative stress and cellular apoptosis of HLEB cells by elevating the rac1-p38 MAPK pathway. Decorin can significantly decrease oxidative stress injury and the proportion of apoptotic HLE cells under exposure to high levels of glucose by suppressing rac1-p38 MAPK pathway activation. Decorin exhibited a protective function against the high-glucose-stimulated oxidative stress and apoptosis. These findings indicate that decorin can be potential treatment to prevent the occurrence of cataract in diabetic patients.

\section{Conclusion}

In brief, decorin decreased oxidative stress injury and the proportion of apoptotic HLE cells in high levels of glucose by suppressing rac1-p38 MAPK pathway activation. Decorin may be a potential drug to prevent cataract development in diabetic patients.

\section{Author Contribution Statements}

The study was designed by Shanshan Du and Fengyan Zhang. The experiments were conducted by Jingzhi Shao. The manuscript was drafted by Jingzhi Shao and Jingjing Wan. Data were analyzed by Jingzhi Shao and Jingjing Wan. Zhang Lirong proofread the document. The authors have granted approval to the final version of manuscript.

\section{Conflict of Interest Statement}

All the authors do not have any possible conflicts of interest.

\section{References}

[1] Haddad NM, Sun JK, Abujaber S, Schlossman DK, Silva PS. Cataract surgery and its complications in diabetic patients. Seminars in ophthalmology. 2014; 29 (5-6): 329-37. Epub 2014/10/18. doi: 10.3109/08820538.2014.959197. PubMed PMID: 25325858.

[2] Jain AK, Lim G, Langford M, Jain SK. Effect of high-glucose levels on protein oxidation in cultured lens cells, and in crystalline and albumin solution and its inhibition by vitamin B6 and $\mathrm{N}$-acetylcysteine: its possible relevance to cataract formation in diabetes. Free radical biology \& medicine. 2002; 33 (12): 1615-21. Epub 2002/12/19. PubMed PMID: 12488130.

[3] Wride MA, Geatrell J, Guggenheim JA. Proteases in eye development and disease. Birth defects research Part C, Embryo today: reviews. 2006; 78 (1): 90-105. Epub 2006/04/20. doi: 10.1002/bdrc.20063. PubMed PMID: 16622853.

[4] Fan H, Sulochana KN, Chong YS, Ge R. Decorin derived antiangiogenic peptide LRR5 inhibits endothelial cell migration by interfering with VEGF-stimulated NO release. The international journal of biochemistry \& cell biology. 2008; 40 (10): 2120-8. Epub 2008/04/01. doi: 10.1016/j.biocel.2008.02.009. PubMed PMID: 18373940.

[5] Lala N, Girish GV, Cloutier-Bosworth A, Lala PK. Mechanisms in decorin regulation of vascular endothelial growth factor-induced human trophoblast migration and acquisition of endothelial phenotype. Biology of reproduction. 2012; $87 \quad$ (3): 59. Epub 2012/06/16. doi: 10.1095/biolreprod.111.097881. PubMed PMID: 22699486.

[6] Grant DS, Yenisey C, Rose RW, Tootell M, Santra M, Iozzo RV. Decorin suppresses tumor cell-mediated angiogenesis. Oncogene. 2002; 21 (31): 4765-77. Epub 2002/07/09. doi: 10.1038/sj.onc.1205595. PubMed PMID: 12101415.

[7] Mohan RR, Tovey JC, Sharma A, Schultz GS, Cowden JW, Tandon A. Targeted decorin gene therapy delivered with adeno-associated virus effectively retards corneal neovascularization in vivo. PloS one. 2011; 6 (10): e26432. Epub 2011/11/01. doi: 10.1371/journal.pone.0026432. PubMed PMID: 22039486; PubMed Central PMCID: PMCPMC3198476.

[8] Ozay R, Turkoglu E, Gurer B, Dolgun H, Evirgen O, Erguder BI, et al. Does Decorin Protect Neuronal Tissue via Its Antioxidant and Antiinflammatory Activity from Traumatic Brain Injury? An Experimental Study. World neurosurgery. 2017; 97: 407-15. Epub 2016/10/17. doi: 10.1016/j.wneu.2016.09.115. PubMed PMID: 27744073.

[9] Davies JE, Tang X, Denning JW, Archibald SJ, Davies SJ. Decorin suppresses neurocan, brevican, phosphacan and NG2 expression and promotes axon growth across adult rat spinal cord injuries. The European journal of neuroscience. 2004; 19 (5): 1226-42. Epub 2004/03/16. doi: 10.1111/j.1460-9568.2004.03184.x. PubMed PMID: 15016081.

[10] Wang S, Du S, Wu Q, Hu J, Li T. Decorin Prevents Retinal Pigment Epithelial Barrier Breakdown Under Diabetic Conditions by Suppressing p38 MAPK Activation. Investigative ophthalmology \& visual science. 2015; 56 (5): 2971-9. Epub 2015/04/02. doi: 10.1167/iovs.14-15874. PubMed PMID: 25829413.

[11] Du S, Wang S, Wu Q, Hu J, Li T. Decorin inhibits angiogenic potential of choroid-retinal endothelial cells by downregulating hypoxia-induced Met, Rac1, HIF-1alpha and VEGF expression in cocultured retinal pigment epithelial cells. Experimental eye research. 2013; 116: 151-60. Epub 2013/09/11. doi: 10.1016/j.exer.2013.08.019. PubMed PMID: 24016866.

[12] Shao JZ, Qi Y, Du SS, Du WW, Li FZ, Zhang FY. In vitro inhibition of proliferation, migration and epithelial-mesenchymal transition of human lens epithelial cells by fasudil. International journal of ophthalmology. 2018; 11 (8): 1253-7. Epub 2018/08/25. doi: 10.18240/ijo.2018.08.02. PubMed PMID: 30140626; PubMed Central PMCID: PMCPMC6090120. 
[13] Cui X, Xie PP, Jia PP, Lou Q, Dun G, Li S, et al. Hsf4 counteracts Hsfl transcription activities and increases lens epithelial cell survival in vitro. Biochimica et biophysica acta. 2015; 1853 (3): 746-55. Epub 2015/01/21. doi: 10.1016/j.bbamcr.2015.01.004. PubMed PMID: 25601714.

[14] Wang GQ, Dang YL, Huang Q, Woo VC, So KF, Lai JS, et al. In Vitro Evaluation of the Effects of Intraocular Lens Material on Lens Epithelial Cell Proliferation, Migration, and Transformation. Current eye research. 2017; 42 (1): 72-8. Epub 2016/06/25. doi: 10.3109/02713683.2016.1156133. PubMed PMID: 27341403.

[15] Rwei P, Alex Gong CS, Luo LJ, Lin MB, Lai JY, Liu HL. In vitro investigation of ultrasound-induced oxidative stress on human lens epithelial cells. Biochemical and biophysical research communications. 2017; 482 (4): 954-60. Epub 2016/11/30. doi: 10.1016/j.bbrc.2016.11.139. PubMed PMID: 27894841 .

[16] Acevedo A, Gonzalez-Billault C. Crosstalk between Rac1-mediated actin regulation and ROS production. Free radical biology \& medicine. 2018; 116: 101-13. Epub 2018/01/14. doi: 10.1016/j.freeradbiomed.2018.01.008. PubMed PMID: 29330095.

[17] Myant KB, Cammareri P, McGhee EJ, Ridgway RA, Huels DJ, Cordero JB, et al. ROS production and NF-kappaB activation triggered by RAC1 facilitate WNT-driven intestinal stem cell proliferation and colorectal cancer initiation. Cell Stem Cell. 2013; 12 (6): 761-73. Epub 2013/05/15. doi: 10.1016/j.stem.2013.04.006. PubMed PMID: 23665120; PubMed Central PMCID: PMCPMC3690525.

[18] Ellenbroek SI, Collard JG. Rho GTPases: functions and association with cancer. Clin Exp Metastasis. 2007; 24 (8): 657-72. Epub 2007/11/15. doi: 10.1007/s10585-007-9119-1. PubMed PMID: 18000759.
[19] Radisky DC, Levy DD, Littlepage LE, Liu H, Nelson CM, Fata $\mathrm{JE}$, et al. Raclb and reactive oxygen species mediate MMP-3-induced EMT and genomic instability. Nature. 2005; 436 (7047): 123-7. Epub 2005/07/08. doi: 10.1038/nature03688. PubMed PMID: 16001073; PubMed Central PMCID: PMCPMC2784913.

[20] Witte D, Bartscht T, Kaufmann R, Pries R, Settmacher U, Lehnert $\mathrm{H}$, et al. TGF-betal-induced cell migration in pancreatic carcinoma cells is RAC1 and NOX4-dependent and requires RAC1 and NOX4-dependent activation of p38 MAPK Oncol Rep. 2017; 38 (6): 3693-701. Epub 2017/10/19. doi: 10.3892/or.2017.6027. PubMed PMID: 29039574.

[21] Veluthakal R, Kumar B, Mohammad G, Kowluru A, Kowluru RA. Tiam1-Rac1 Axis Promotes Activation of p38 MAP Kinase in the Development of Diabetic Retinopathy: Evidence for a Requisite Role for Protein Palmitoylation. Cellular physiology and biochemistry: international journal of experimental cellular physiology, biochemistry, and pharmacology. 2015; 36 (1): 208-20. Epub 2015/05/15. doi: 10.1159/000374065. PubMed PMID: 25967961; PubMed Central PMCID: PMCPMC4435616.

[22] Rastogi R, Geng X, Li F, Ding Y. NOX Activation by Subunit Interaction and Underlying Mechanisms in Disease. Frontiers in cellular neuroscience. 2016; 10: 301. Epub 2017/01/26. doi: 10.3389/fncel.2016.00301. PubMed PMID: 28119569; PubMed Central PMCID: PMCPMC5222855.

[23] Du S, Shao J, Xie D, Zhang F. Decorin inhibits glucose-induced lens epithelial cell apoptosis via suppressing p22phox-p38 MAPK signaling pathway. PLoS One. 2020 Apr 27; 15 (4): e0224251. doi: 10.1371/journal.pone.0224251. PMID: 32339204; PMCID: PMC7185589. 